Jenny Carlsson*, Magnus Heldin, Per Isaksson and Urban Wiklund

\title{
Investigating tool engagement in groundwood pulping: finite element modelling and in-situ observations at the microscale
}

https://doi.org/10.1515/hf-2019-0133

Received May 8, 2019; accepted October 2, 2019; previously published online November 16, 2019

\begin{abstract}
With industrial groundwood pulping processes relying on carefully designed grit surfaces being developed for commercial use, it is increasingly important to understand the mechanisms occurring in the contact between wood and tool. We present a methodology to experimentally and numerically analyse the effect of different tool geometries on the groundwood pulping defibration process. Using a combination of high-resolution experimental and numerical methods, including finite element (FE) models, digital volume correlation (DVC) of synchrotron radiation-based X-ray computed tomography (CT) of initial grinding and labscale grinding experiments, this paper aims to study such mechanisms. Three different asperity geometries were studied in FE simulations and in grinding of wood from Norway spruce. We found a good correlation between strains obtained from FE models and strains calculated using DVC from stacks of CT images of initial grinding. We also correlate the strains obtained from numerical models to the integrity of the separated fibres in lab-scale grinding experiments. In conclusion, we found that, by modifying the asperity geometries, it is, to some extent, possible to control the underlying mechanisms, enabling development of better tools in terms of efficiency, quality of the fibres and stability of the groundwood pulping process.
\end{abstract}

Keywords: CT, defibration, DVC, FE, grinding, wood

\footnotetext{
*Corresponding author: Jenny Carlsson, The Ångström Laboratory, Department of Engineering Sciences, Uppsala University, Box 534, SE-751 21 Uppsala, Sweden, e-mail: jenny.carlsson@angstrom.uu.se. https://orcid.org/0000-0003-3950-8840 Magnus Heldin, Per Isaksson and Urban Wiklund: The Ångström Laboratory, Department of Engineering Sciences, Uppsala University, Box 534, SE-751 21 Uppsala, Sweden
}

\section{Introduction}

In groundwood pulping, both the efficiency of the process and the quality of the product depend on the mechanisms occurring in the contact between the wood and the tool. Lately, processes which rely on designed grit surfaces for grinding have been developed and are being used commercially. Significant progress has been made in the production of controlled grinding tools. With improved control in design of such surfaces, an understanding of the fundamental grinding mechanisms is increasingly important in order to design new, more efficient tools. However, most previous research on the fibre separating mechanisms in groundwood pulping has focused on fatigue and peeling of fibres due to compressive and shearing forces (Atack and May 1962; Salmén and Fellers 1981), most often on a larger scale than the actual geometries of the grinding tool or the fibres themselves. This is a consequence of the assumption that fatigue pre-treatment followed by fibre peeling are necessary mechanisms when separating the fibres, whereas less energy is required if the fibres can be separated without prior fatigue. However, to understand the other contributing mechanisms that assist in fibre separation, more detailed studies of the interaction between the individual fibres and the tool geometry are needed. Studies on the interactions close to the interface between wood and tool are scarce (Dornfeld 1981; Tuovinen et al. 2009; Tuovinen and Fardim 2015). The present work aims to study the local interaction between the wood structure and asperity geometries, well controlled and with an overall size on the scale of the fibre width. Lab-scale grinding experiments are combined with finite element (FE) modelling to gain knowledge of the interactions occurring in the groundwood pulping process.

Groundwood pulping is a mechanical pulping process in which logs of wood are pressed against a rotating pulpstone in order to separate the individual fibres and thereby create pulp. Traditionally, the pulpstone is manufactured as a composite with hard particles embedded in a ceramic matrix in a random manner. Before use, the surface of the stone is burred and conditioned to give it a somewhat wavy profile, with exposed and protruding 
particles, Figure 1a. This means that the tool surface has geometries on different scales; an overall cylindrical shape, about a meter in radius, a surface waviness with an amplitude and wavelength in millimetres and a microroughness with edges, remaining from the conditioning, measured in tens of micrometres and even smaller than that. All these geometries influence the interaction with the wood. The larger two are responsible for the largescale fatigue. Such fatigue processes have been studied experimentally both in global and flat compression and shearing (Uhmeier and Salmén 1996; De Magistris and Salmén 2005) and in tool surfaces sliding on wood (Atack and May 1962; Enström et al. 1990; Sandås and Lönnberg 1990; Sandås 1991; Björkqvist et al. 2007; Salmi 2012). On a larger scale, the stress distribution possibly leading up to fatigue in a cellular material (representing wood) as an uneven countersurface is sliding on it has been studied both experimentally and analytically (Höglund and Tistad 1973).

The micro-roughness can also cause fatigue, although the localisation of the stress renders its effect to be very superficial. Due to the random nature and high variability of the micro-roughness of a traditional pulpstone, micro-roughness is often considered detrimental, as fibres are easily fractured by the stochastic contacts with sharp grits. Most fibre shortening and splitting in industrial defibration is believed to occur due to grits damaging the fibres during the initial, fatigue-based, defibration when the fibres are still attached to the wood (Reme 2000). Both Atack and May (1958) and Dornfeld and $\mathrm{Wu}$ (1977) concluded that, given the proper largescale geometries of the stone surface, an unconditioned surface has very high micro-roughness and too many sharp edges to produce good pulp. Attempts to completely avoid micro-roughness have been reported by Atack and May (1962) who made early tests with steel tools totally lacking geometries smaller than $0.1 \mathrm{~mm}$. Using these they could separate fibres, apparently solely due to fatigue, although they concluded that the tool was ineffective and unpractical. However, the micro-roughness is small enough to interact directly with individual fibres, and apart from inducing superficial fatigue, it has the important and more obvious role of peeling fibres from the wood, Figure $1 \mathrm{~b}$.

In the recent years, grinding tools with a more controlled surface topography have been developed and are being used industrially. These surfaces are based on a single layer of diamond grits carefully distributed over the surface, instead of a large number of particles embedded in a matrix, resulting in a surface with much smaller variability, and have shown significant improvements in the energy consumption as well as production rate of the process (Lucander et al. 2010; Tuovinen 2012, 2016, 2018; Tuovinen and Fardim 2015). Even more recently, surfaces with extended microscale ridges with extreme control in distribution, shape, orientation and height have been produced (Heldin and Wiklund 2019) that promise to alter the fine-scale separation mechanisms. Further improvement could be obtained if the surfaces could be optimised for fibre separation. However, the mechanisms involved in micro-roughness fibre separation have proven hard to study separately as they are normally swamped by other mechanisms caused by the larger geometries, and controlled studies are essentially hindered by the stochastic height and distribution of asperities in the pulpstones (Tuovinen and Fardim 2015). There is thus a large untapped potential in newer tools that rely less on large-scale fatigue for defibration and instead promote rapid and clean fibre separation utilising controlled micro-roughness, meaning that the energy consumption could be reduced without increasing the amount of fibre damage. Designing the tool surface to have a controlled distribution and height of asperities directly influences the localisation of stresses and thus the possibilities of superficial fatigue and fibre release. There is, however, still a large gap between theoretical
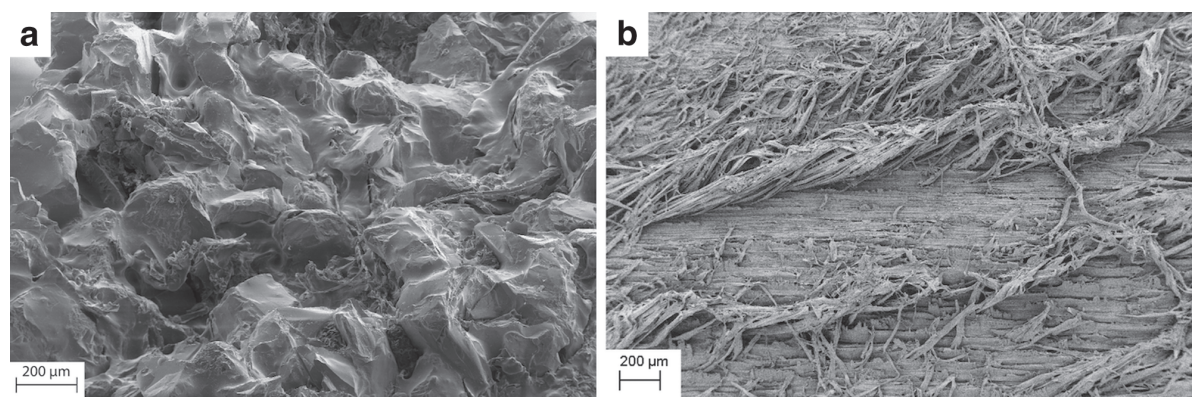

Figure 1: SEM micrographs of the main actors of the groundwood pulping process.

(a) a conventional grinding stone and (b) worn wood surface with fibres being separated and peeled from the surface. 
consideration regarding the shape and distribution of asperities, and the actual outcome of the grinding process. If the mechanisms involved in the fibre separation can be better understood and predicted, it is possible to design even better grinding surfaces than the currently available ones.

The orientation of the wood with respect to the stone axis has been shown to influence the character of the produced fibres, with higher degrees of shorter fibres and fines when the angle between the stone axis and the wood structure increases (Beath 1958; Heinemann et al. 2016; Saharinen et al. 2016). Such angular alignment is also possible to influence using designed tool surfaces.

In order to investigate the effect of such small geometries in isolation, we turn to numerical models. FE models are frequently used in applications of, for example, cutting, forming and turning to study and improve the mechanics of the process. Studies using numerical models to investigate the mechanical response of the fibre in the pulping process are however scarce. Previous research has focused on the combination of compression and shear, without considering the mechanisms causing this loading (Nairn 2006; De Magistris and Salmén 2008; Fortino et al. 2015). Using a high-resolution FE model, De Magistris and Salmén (2008) reported numerically predicted deformations well in agreement with ones observed in a scanning electron microscope (SEM). In a similar study, Fortino et al. (2015) reported stresses in the different layers of the cell wall. An interesting observation is that, although the load cases considered consisted of a combination of global compression and shear, there are large local tensile principal stresses in the direction transverse to the cell wall, which suggest a mechanism for fibre separation under compression loads.

While providing interesting observations with respect to the deformation and stresses of a deformed microstructure, neither of these studies considered the mechanisms causing this loading, i.e. the tool interaction. On the other hand, treating wood as a continuum and instead focusing on the loading mechanisms, accurate global fracture paths have been predicted by, for example, Holmberg et al. (1999). In an attempt to bridge the gap between high-resolution cell models and continuum models, Isaksson (2015) applied a gradientenhanced elasticity continuum theory, which was shown to better capture the strain fields of wood under an indenter (see also the work by Heldin et al. 2016). This method is not, however, suited to study the deformation and strain of the individual cells under an indenter, as such displacements occur in a scale below. For this, high-resolution models are needed.

\section{Materials and methods}

FE modelling of initial contacts: FE simulation of the indentation process was performed using the commercial code Abaqus (2015). A simplified model of softwood clear-wood (i.e. knot-free, nontension/compression wood) was used. In this model, only two constituents of wood are considered, the secondary (S) wall (which is considered to consist of the $S_{1}, S_{2}$ and $S_{3}$ layers) and the compound middle lamella (CML), which is considered to consist of the middle lamella and the primary wall. Each of these constituents was modelled as an orthotropic, linearly elastic continuum (using the engineering constants option in Abaqus), i.e.

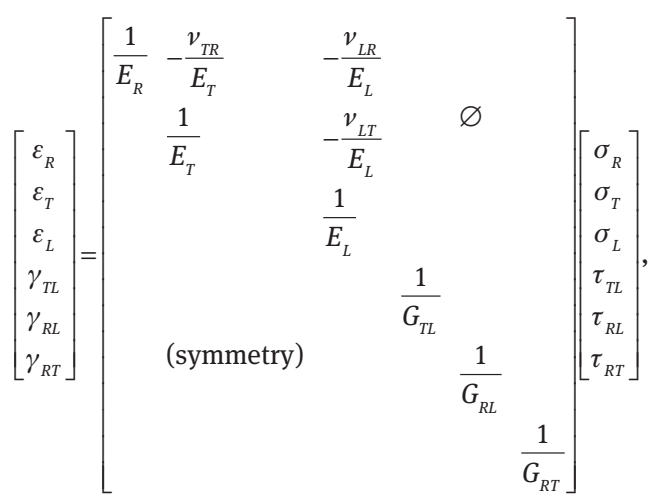

where $\varepsilon_{R / T / L}$ are the normal strains in the radial $(R)$, tangential $(T)$ and longitudinal ( $L$ ) directions of the wood stem, and $\gamma_{T L / R L / R T}$ are the (engineering) shear strains while $\sigma_{R / T / L}$ and $\tau_{T L / R L / R T}$ are the corresponding normal and shear stresses. The elasticity parameters $E_{R / T / L}, v_{L R / L T / R T}$ and $G_{L R / L T / R T}$ are the Young's moduli, Poisson's ratio and shear moduli, corresponding to the same directions adapted from primarily Harrington et al. (1998), Table 1. Due to the similarity of the mechanical properties in the $R$ and $T$ directions, the local (wood cell) properties presented by Harrington et al. apply to the larger scale of the whole model. In the results, the transverse direction (relative to the indentation loading) is referred to as $R$ in order to comply with experiments, but it should be noted that the results are just as valid if this direction is the tangential direction. No influence of micro-fibril angles, etc. was considered. The material properties reported by Harrington et al. are for room-temperature wood with $12 \%$ moisture content, whereas pulping typically occurs at elevated temperature and moisture levels. The authors have not been able to find any studies reporting material properties of the cell wall constituents for these conditions. [Influence of moisture and heat on the global material behaviour of wood has been studied by, for example, Moilanen et al. (2017) but not on this scale.]

Table 1: Elastic constants for the two constituents of the cell wall.

\begin{tabular}{lrrrrrrrrr}
\hline & $E_{L}$ & $E_{R}$ & $E_{T}$ & $\boldsymbol{v}_{L R}$ & $\boldsymbol{v}_{L T}$ & $\boldsymbol{v}_{R T}$ & $\boldsymbol{G}_{L R}$ & $\boldsymbol{G}_{L T}$ & $\boldsymbol{G}_{R T}$ \\
\hline $\mathrm{S}$ & 60 & 9 & 9 & 0.1 & 0.1 & 0.4 & 3 & 3 & 3 \\
$\mathrm{CML}$ & 20 & 5 & 5 & 0.1 & 0.1 & 0.3 & 2 & 2 & 2 \\
\hline
\end{tabular}

Moduli are given in GPa. 
A small, repeated unit consisting of the wood under one asperity was analysed. The geometry consisted of $7 \times 4$ wood “cells”, Figure $2 \mathrm{~b}$. The wood was assumed to be perfectly periodic and each wood cell was assumed to have edges $50 \mu \mathrm{m}$ long. The relative density (defined as the proportion of solid volume to the total volume) of the wood model is 0.4 , which is close to the average relative density of Norway spruce. The depth of the model (in the length direction of the cells) was $175 \mu \mathrm{m}$. Symmetry boundary conditions were applied to the front and back sides, and the displacements of the right and left sides were constrained to follow each other (tied), in order to mimic a repetitive behaviour. The top side was free, and the bottom side was clamped. The wood structure was meshed with around 430000 linear hexahedral elements, each having an edge length of roughly $2.5 \mu \mathrm{m}$.

Three different asperity geometries were studied, Figure 2a, all modelled as rigid pyramids with apex angle $90^{\circ}$ : one with a small tip radius $(10 \mu \mathrm{m})$, one with a larger tip radius $(30 \mu \mathrm{m})$ and one with a truncated tip (top side diameter $40 \mu \mathrm{m}$ ). The asperities were meshed with around 12000 linear elements, primarily quadrilateral elements, having about $2 \mu \mathrm{m}$ edge lengths. The contact between the asperity and surface was a penalty type contact with a Coulomb friction coefficient of 0.3 .

In a first simulation, the pyramid was indented to a depth of half the cell height to simulate stress and strain distribution during relatively large deformations. In a second simulation, the pyramid was indented only a fraction of the cell height $(5 \mu \mathrm{m})$, then moved horizontally (equivalent to, for example, radial sliding) a distance equal to the width of one cell, in order to simulate how the stress and strain distribution might vary during grinding.

Tomography of initial contacts: The initial interaction between the tool and a wood sample was also studied using synchrotron-based X-ray computed tomography (CT) at the TOMCAT beamline at the Swiss Light Source, PSI, Switzerland. The setup, consisting of a small compression-based tester with a specially designed polymeric [polylactic acid (PLA)] tool holder, allowed for simultaneous sliding and loading between the tool and the wood, Figure 3. The tool consisted of a $5 \times 5 \mathrm{~mm}^{2}$ surface with asperities in the form of small diamond pyramids distributed over the surface. Using an energy of $16 \mathrm{keV}$, 1201 radiographs, having a pixel size of $3.25 \mu \mathrm{m}$, were captured while simultaneously rotating the sample $180^{\circ}$. Before a new set of radiographs were captured, the tool was slid a few tens of micrometres relative to the wood surface, in the radial direction of the wood, making the tool move both along the wood surface and slightly towards the wood. Sets of radiographs were captured before, during and after initial engagement, and a few times with the tool in contact. No relative motion occurred during the actual scans. Image stacks were obtained from the recorded radiographs using a reconstruction algorithm (Marone and Stampanoni 2012), as exemplified in Figure 4.
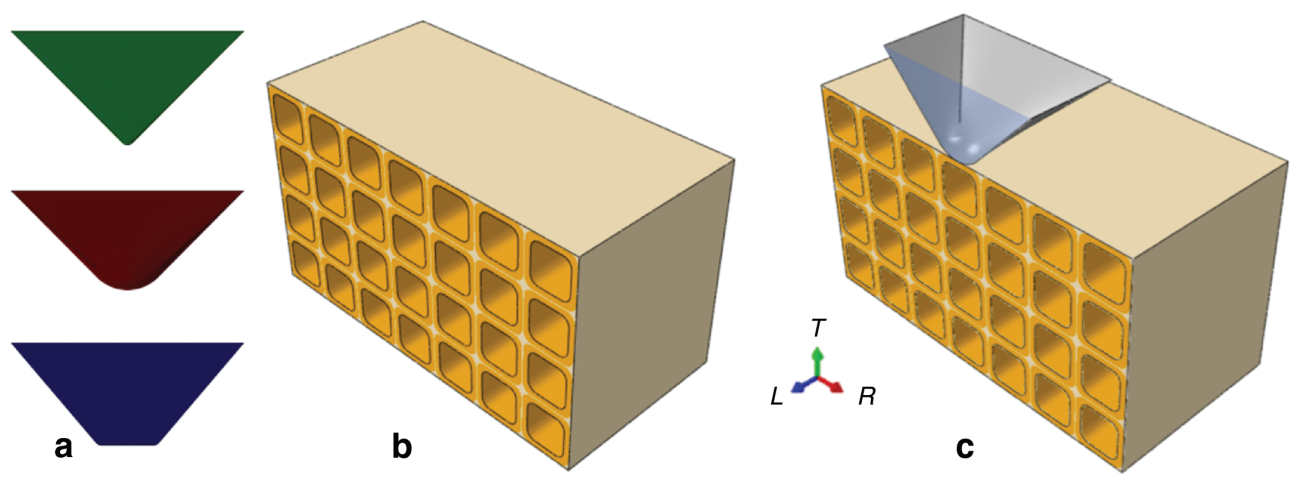

Figure 2: Finite element model.

(a) pyramid-shaped asperity geometries, (b) the wood structure consisting of the S layer and CML and (c) the full model including the global coordinate system.
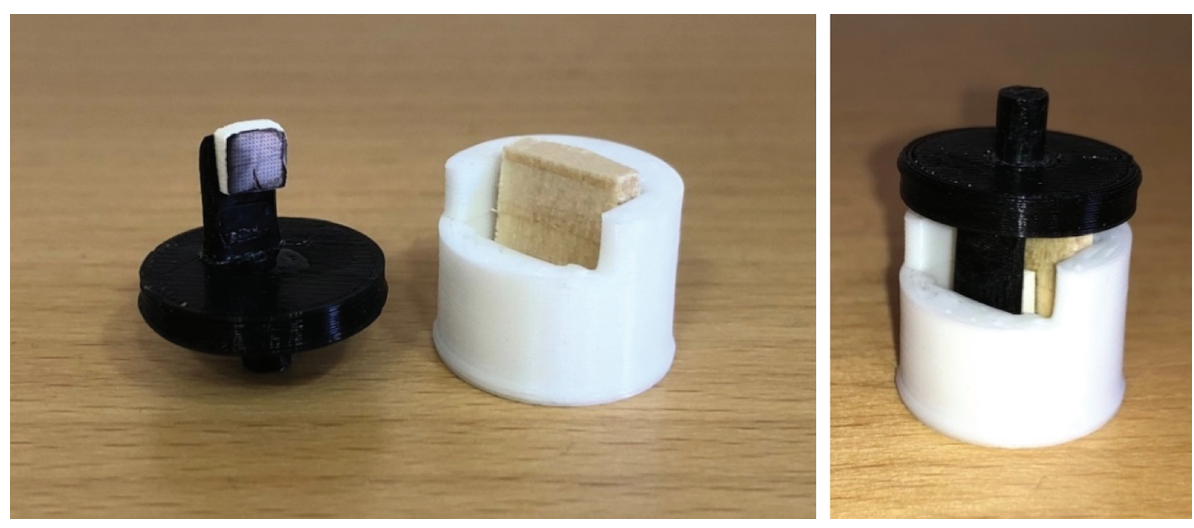

Figure 3: Experimental setup for X-ray CT of initial contact.

The vertical arm of the black tool holder has a shiny tool mounted (diamond pyramids are barely visible). The white holder has a piece of wood mounted. As the black holder is pressed into the white holder, its arm is forced to bend and the tool is moved towards (into) the wood. 


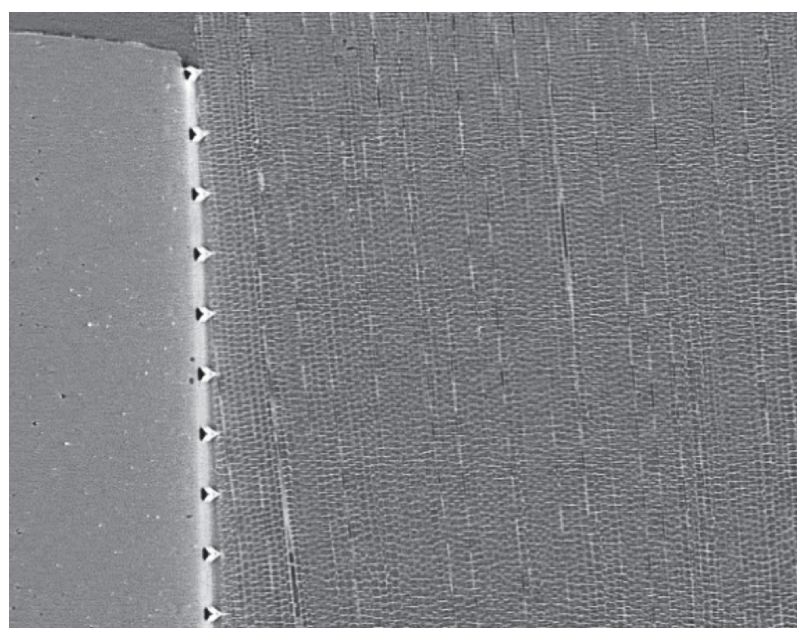

Figure 4: Reconstructed CT image showing the diamond tool (left) and wood with its cellular structure edge on.

Ten diamond pyramids are visible in this section. At this particular step, the diamonds have made contact with the wood and deformed the wood structure slightly.

DVC of CT data: A digital volume correlation (DVC) algorithm (van Dijk et al. 2019), based on a higher-order FE discretisation, was used to estimate the deformation fields in the wood. The algorithm tracks the position of material points in two subsequent image stacks by cross-correlating unique greyscale distributions in the CT stacks. To increase the sensitivity of the cross-correlation to local differences, the volume is divided into a set of sub-volumes, each containing unique greyscale patterns, which are traced in the deformed volume. By tracing these patterns in the deformed volume, displacements of the sub-volumes and, by means of interpolation, also the global displacement field are estimated. Displacements are estimated on the sub-voxel level. Essentially, the algorithm solves an optimisation problem where the global displacement field minimises an objective function using a regularisation technique in connection to the correlation. Regularisation is added to the correlation process to decrease sensitivity to noise and artefacts and to compensate for very little variation in the greyscale distribution in the sub-volumes. In this study, a sub-volume size of $40 \times 40 \times 40$ voxels and regularisation parameter of magnitude 100 (see van Dijk et al. 2019 for details) provided a good balance between accuracy in the estimated displacement field and computational cost for the considered wood material.

Grinding experiments: Experiments with repeated sliding were performed to illustrate the initial damage mechanisms as the grinding tool works on the wood. A diamond grinding tool was now mounted on a stationary tool holder and loaded with $10 \mathrm{~N}$ against a flat wood surface submerged in a water bath at $95^{\circ} \mathrm{C}$. While in contact with the tool, the wood specimen was moved $15 \mathrm{~mm}$ in the radial direction, perpendicular to the fibre direction, the movement taking about $1 \mathrm{~s}$. The tool was then lifted from the wood surface and the wood sample was returned to the starting position. The process was repeated 20 times. Afterwards, both the worn surface and the tool surface were imaged in an SEM.

\section{Materials \\ Wood}

In all experiments, the samples used were made from Norway spruce [Picea abies (L.) H. Karst], which is the most commonly used wood species in Swedish pulping today. The samples came from an approximately 50-year-old tree, grown in the middle of Sweden. Typical cell diameters of the wood are in the range of $20-50 \mu \mathrm{m}$ and the relative density varies between 0.2 and 0.5 over the annual rings. To preserve the wood, log pieces were stored in a freezer in the green state and cut to the dimensions used in the experiments after thawing. In the tomography experiments, the wood used was dry, as this was considered to be the best way to keep the properties stable over the entire experiment. In the grinding experiments, the wood was submerged in $95^{\circ} \mathrm{C}$ water.

\section{Diamond grinding surfaces}

Well-defined grinding surfaces were manufactured as diamond films deposited on patterned mould surfaces (Gåhlin et al. 1999; Heldin and Wiklund 2019). The position and size of the pyramid-shaped asperities were controlled in the micrometre range. In this study, three different pyramid geometries were used, small and sharp (base side length $50 \mu \mathrm{m}$ ), slightly larger with a small truncated top (base side length $125 \mu \mathrm{m}$, top side length $5 \mu \mathrm{m}$ ) and pyramids with truncated tops (base side length $150 \mu \mathrm{m}$, top side length $40 \mu \mathrm{m}$ ), Figure 5 . In the tomography experiments, only the smallest pyramid geometry was used. All three pyramid geometries were used in the grinding experiments.

\section{Results}

\section{FE modelling}

To get a notion of the amount of tensile (separating) strains, first principal normal strains, i.e. the highest occurring
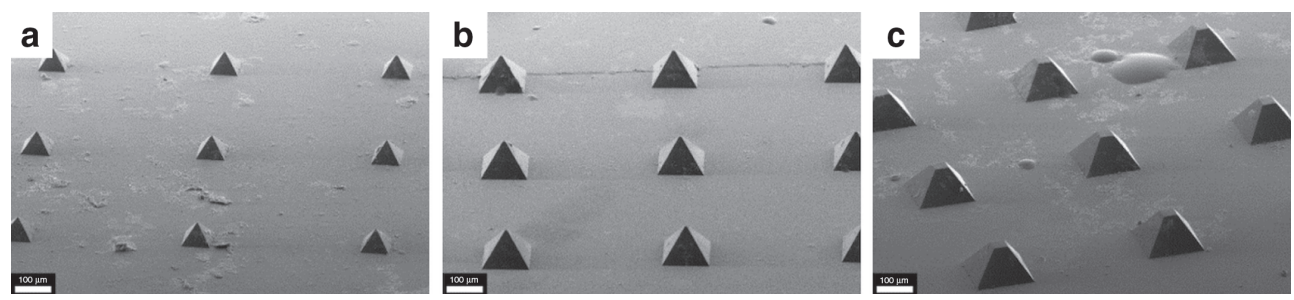

Figure 5: SEM micrographs of the diamond grinding surfaces.

(a) small pyramids (base side length $50 \mu \mathrm{m}$ ), (b) larger pyramids (base side length $125 \mu \mathrm{m}$, top side length $5 \mu \mathrm{m}$ ) and (c) pyramids with truncated tops (base side length $150 \mu \mathrm{m}$, top side length $40 \mu \mathrm{m}$ ). 
tensile strain, obtained from the FE model for pure indentation to $25 \mu \mathrm{m}$ (no sliding) are shown in Figure 6. For the small pyramid radius, Figure $6 \mathrm{a}$, the strains extend to a small depth, less than one cell diameter. For the larger radius, Figure $6 \mathrm{~b}$, and even more so for the truncated pyramid, Figure $6 \mathrm{c}$, the strains extend deeper. Even so, the difference in highest principal normal strain is small between the models. [While the first principal normal strain is positive (i.e. tensile), it should be noted that the magnitude of the third principal normal strain, which is compressive, is larger than the magnitude of the first principal normal strain.]

The mechanism of fibre separation is dependent on deformation in the CML. Tensile strain in the radial direction $\left(\varepsilon_{R}\right)$ is present in the vertical CMLs on either side of the indenters, Figure 7a. In the loading direction, the normal strain $\left(\varepsilon_{T}\right)$ is mainly compressive (Figure $7 \mathrm{~b}$ ), but the shear strain $\left(\gamma_{R T}\right)$ is large in the region directly beneath and in a few cells to either side of the indenters, Figure 8. Combined, these observations indicate that cells are likely to be peeled apart upon (repeated) loading.

The simulation with the sliding pyramid (indentation depth $5 \mu \mathrm{m}$ ) affirms the results of the pure indentation simulation. For the pyramid with a small radius, tensile strain is localised to the surface. For the pyramid with a larger radius, Figure 9a, the tensile strain extends to the next layer and with the truncated pyramid we see tensile strains further down in the structure. This applies to all instances of the motion, as well as to the maximum principal shear strain $\gamma_{\max }$ (visualised in the time instance when the pyramid is directly above the vertical cell wall) Figure 9b. The vertical CML has tensile $\varepsilon_{R}$ strains during the full sliding motion, Figure 10a. Note that the pyramid moves over the CML in question. The highest tensile $\varepsilon_{R}$ strains occur when the pyramid is directly above the vertical cell wall; this is also when the highest compressive $\varepsilon_{T}$ strains occur. The shear strain $\gamma_{R T}$ also takes on its maximum value as the pyramid is directly above the vertical cell wall, Figure 10b. This variation in strain can be related to the development of superficial fatigue.

The force required to indent the truncated pyramid a distance equal to half a cell size is significantly higher than the force required to indent a sharp pyramid the same distance. If instead the force is kept constant, the depth at which a particular strain level occurs is similar for all three pyramids, while the maximum principal strain is smaller for the pyramid with a large radius and especially for the truncated pyramid.
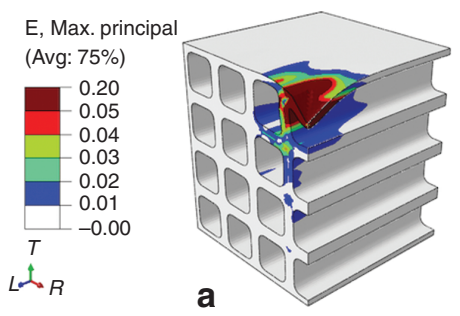
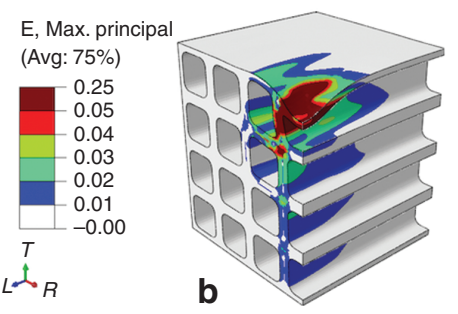

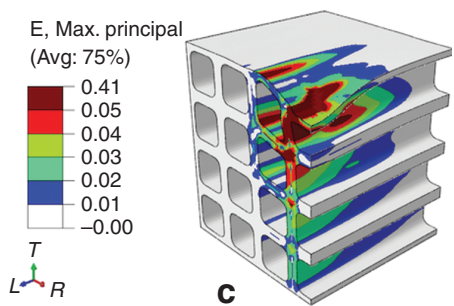

Figure 6: First principal strains from FE modelling of indentation.

(a) pyramids with a small radius and (b) a large radius and (c) truncated pyramids. The pyramid itself is removed from the images, but placed directly above the cut corner, i.e. right above a row of cells.
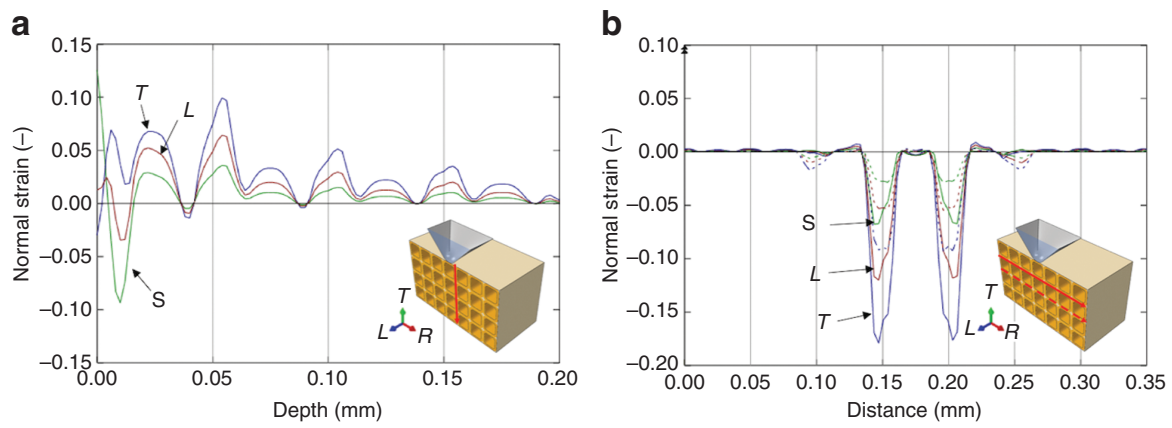

Figure 7: Normal strain in the middle lamella.

(a) variation of $\varepsilon_{R}$ vs. depth plotted along the vertical cell wall to the right of the pyramid (along the path indicated by the red arrow) and (b) variation of $\varepsilon_{T}$ vs. distance in the $R$ direction plotted along the horizontal cell wall one and two rows down (at 50 and $100 \mu \mathrm{m}$ depth, along the path indicated by the red arrow). In all figures, red lines represent pyramids with a large radius ( $L$ ), green lines represent pyramids with a small radius (S) and blue lines represents truncated pyramids ( $T$ ). Dashed lines represent the second layer of cells. 
a

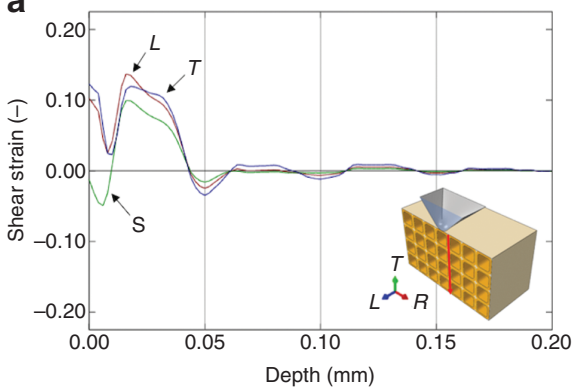

b

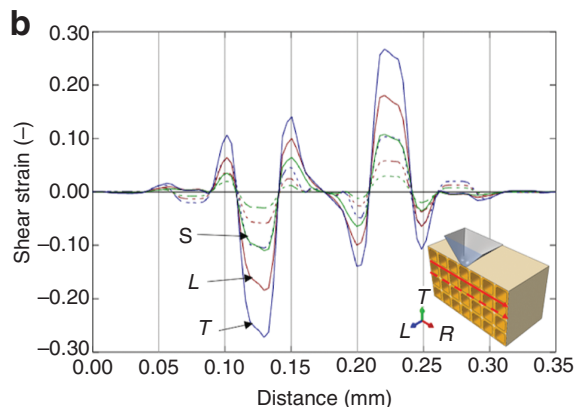

Figure 8: Shear strain in the middle lamella.

(a) variation of $\gamma_{R T}$ vs. depth plotted along the vertical cell wall to the right of the pyramid (along the path indicated by the red arrow) and (b) variation of $\gamma_{R T}$ vs. distance in the $R$ direction plotted along the horizontal cell wall one and two rows down (at 50 and $100 \mu \mathrm{m}$ depth, along the path indicated by the red arrow). In all figures, red lines represent pyramids with a large radius ( $L$ ), green lines represent pyramids with a small radius (S) and blue lines represent truncated pyramids $(T)$. Dashed lines represent the second layer of cells.
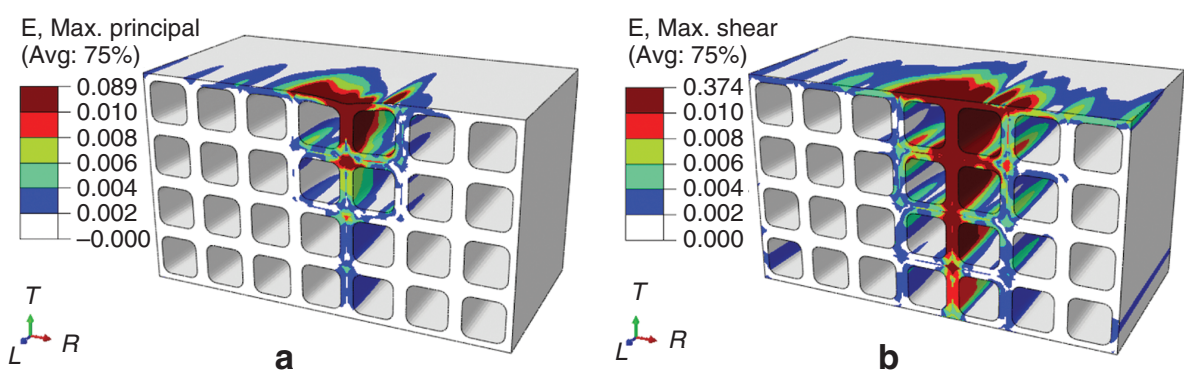

Figure 9: Strains during sliding motion of the large-radius pyramid.

(a) maximum principal strain and (b) maximum shear strain, $\gamma_{\max }$. The pyramid is removed from the images, but is located straight above the vertical cell wall. Indentation depth $5 \mu \mathrm{m}$, sliding direction is radial, to the right.

a

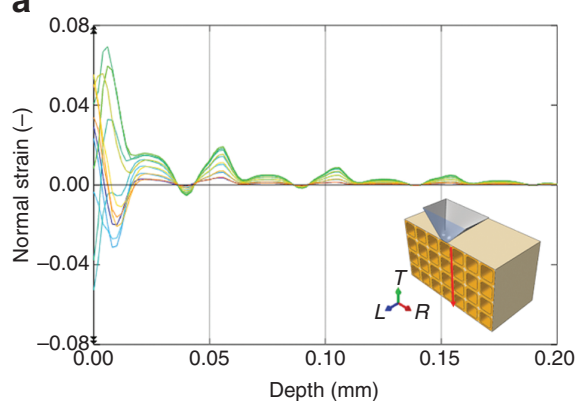

b

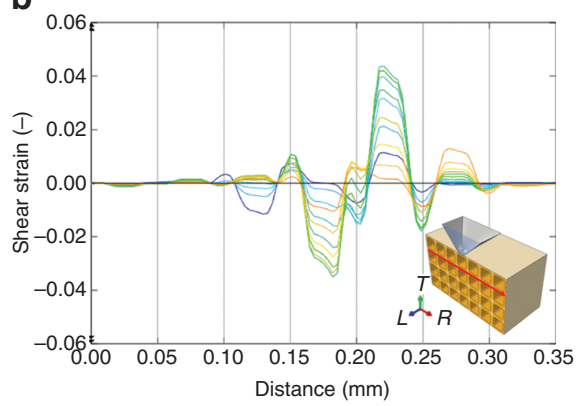

Figure 10: Strains in the middle lamella during 11 instances of the sliding motion (indentation depth $5 \mu \mathrm{m}$ ), from the centre of the middle-most cell to the centre of the cell to the right of it, of the large-radius pyramid.

(a) normal strain $\varepsilon_{R}$ vs. depth plotted along the vertical cell wall to the right of the initial position of the pyramid (along the path indicated by the red arrow) and (b) shear strain $\gamma_{R T}$ vs. distance in the $R$ direction plotted along the horizontal cell wall one row down (at $50 \mu \mathrm{m}$ depth, along the path indicated by the red arrow). In both figures, the strain at different indenter positions is indicated using a colour spectrum; blue represents the initial position, followed by light blue, etc. and finally orange represents the final position.

\section{DVC of CT data}

Displacement from DVC of the grinding tool in contact with wood is shown in Figure 11. Red colour represents regions with large displacements and blue colour represents regions with small or no displacement. We see that for a volume portion under nine pyramid-shaped asperities, vertical displacements are localised to a small volume near the surface, in particular to the volumes in the proximity of the asperities. Figure 12 displays principal (finite) 
strains in the wood obtained by numerical spatial differentiation of the displacement field. Again, red colour represents areas with high strain and blue colour represents areas with small or no strain. The largest tensile strain is located to the region close to the indenter, and the volume of high tensile strain extends only about one cell diameter underneath the indenter.

\section{Wood surface after grinding experiment}

The worn wood surfaces look very different after the scratching experiments, Figure 13. The wood surfaces which have been scratched with the small and the large pyramids show distinct parallel grooves and fibres are cut transversely rather than being detached. The wood which has been scratched with the truncated pyramids has a very different appearance, showing long fibres being detached.

The extracted fibres confirm this observation. For the sharp pyramids, the detached fibres have been cut into short segments, of lengths similar to the distance between the rows of pyramids in the tool surface used. For the
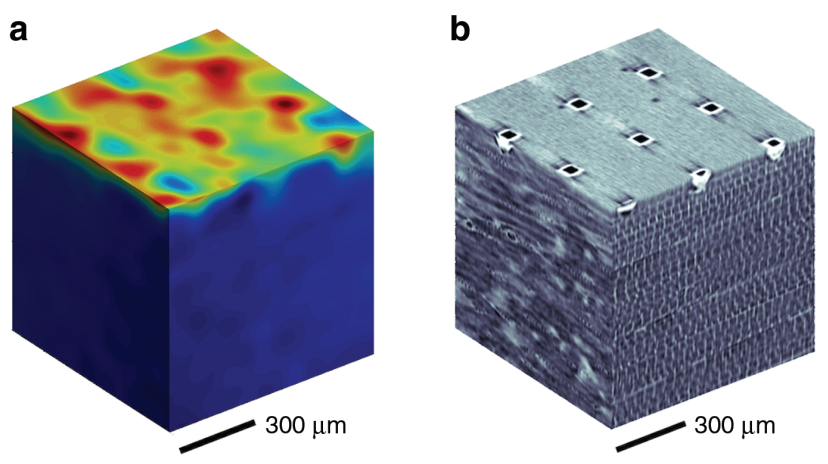

Figure 11: Displacement from DVC of CT data under an area containing nine pyramids.

(a) displacement in vertical (global $T$ ) direction and (b) visualisation of the volume showing the diamond pyramids and the wood. truncated pyramids, the extracted fibres are longer, and there is no obvious relation between the fibre length and the distance between the pyramids.

\section{Discussion}

Despite the obvious differences between the different methods with respect to loading, material, etc., the correlation between the results of the different methods is surprisingly good. Both FE and DVC of the CT of initial grinding show very local deformation and shallow strain fields for the smaller pyramid radius. This trend will hold also for some variation in material stiffness or microstructural architecture. Such extreme local deformation is likely to promote local damage and fibre fracture rather than fibre detachment. Affirmatively, in the grinding experiment, we find that the sharper pyramids tend to cut the fibres into short segments, of lengths similar to the distance between the rows of pyramids in the tool surface, and the worn wood surface shows distinct parallel scratch marks. These observations combined indicate that for small or sharp asperities, deformation and consequently also damage are extremely localised. As a consequence, instead of detaching fibres, repeated sliding of the pyramids cut the fibres transversely along the tracks.

FE models of indentation with a larger pyramid radius, and especially with the truncated pyramid, show larger deformation fields and tensile strains extending deeper down into the material. Or correspondingly, if the loading force is kept constant rather than the indentation depth, the depth at which a particular strain level occurs is similar, but the highest strains have lower magnitudes for the large radius and truncated pyramid. We are thus less likely to damage or cut fibres and more likely to detach long and undamaged fibres. This is supported by the fact that in the grinding experiments, primarily when using the truncated pyramids, the extracted fibres are longer and not cut like the fibres from the experiments using sharp pyramids.
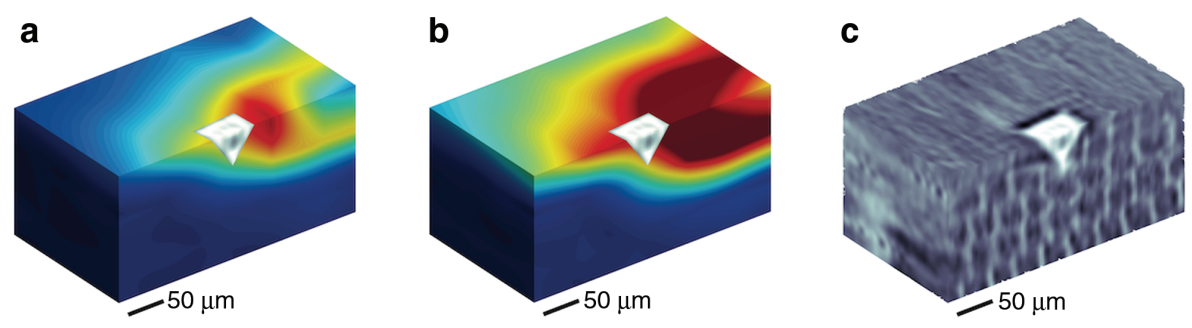

Figure 12: Strain from DVC of CT data of a small volume underneath one pyramid.

(a) first principal strain, (b) maximum shear strain, $\gamma_{\max }$ and (c) visualisation of the volume showing the diamond indenter (white) and individual cells of the wood. For illustrative purposes, the pyramid has been added to the strain visualisations. Pyramid sliding direction is to the left. 

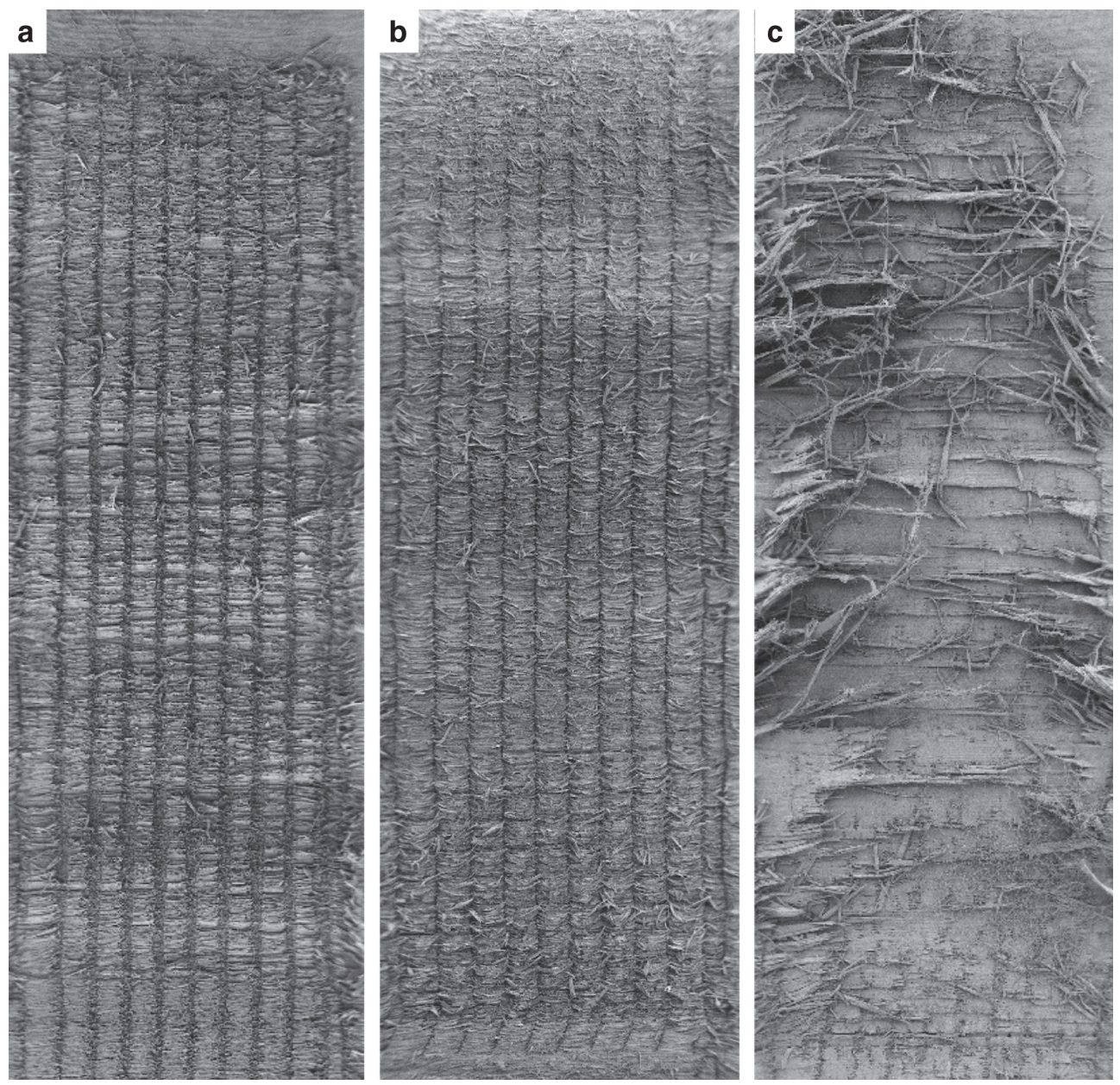

Figure 13: SEM micrographs of the 15-mm-long wear marks after sliding tests in the radial direction using: (a) small pyramids, (b) large pyramids and (c) truncated pyramids.

The tool has been sliding upwards in the images.

In the FE models, the localisation of the strains inversely correlates to the radius of the indenting pyramid; the larger the contact area, the less localised are the strains, meaning that the strains extend deeper into the wood for a given indentation depth, or that the maximum strain is smaller for a given indentation depth. Both the experiments and the numerical models show that the sharp pyramids used are too extreme, both in size and radii, to successfully detach long fibres, whereas the truncated pyramids seem more promising. For the latter, it is interesting to see that a large number of long fibres are detached already after such a short test. This indicates that, using grinding tools with controlled asperities of dimensions close to those of the wood cells, it is possible to detach fibres using local, superficial low-cycle fatigue. Hence, extensive fatigue including thousands of encounters speculated to be necessary (Salminen et al. 2014) might not be a prerequisite for fibre release with this kind of tool geometry.
The different conditions under which the different experiments in this study have been performed are an obvious limitation. Due to lack of better data, the material model in the FE simulations is based on dry wood at room temperature, although it is well known that wet and hot wood differs significantly from dry wood. In addition, synchrotron radiation dries and deteriorates the material. However, the primary purpose of the FE and CT experiments is to see the distribution of stresses and strains in the material. The distribution of the strains around the different asperities will hold for a large variation in material stiffness or microstructural architecture, meaning that the comparisons are nonetheless relevant. The scratching experiments are performed under more realistic conditions, as the desired outcome is not elastic deformation but fibre separation. Thus, they also differ significantly from the other two. Still, the correlation between the different methods suggests that 
despite these shortcomings, the results are valid and the methodology is promising.

\section{Conclusions}

A methodology for experimentally and numerically analysing the effect of different asperity geometries on the groundwood pulping defibration process has been presented. We are able to correlate the strain fields obtained from numerical models to the observations in lab-scale grinding experiments, specifically to the integrity of the separated fibres. This correlation means that the combination of high-resolution experimental and numerical methods offers great possibilities to investigate both the actual tool to wood contact and to study how different grinding surface designs influence the defibration process. By modifying the asperity geometries, it is, to some extent, possible to control the underlying mechanisms to a higher degree than in the current state-of-theart processes, thereby continuing the development of groundwood pulping towards a situation where microroughness separation rather than large-scale fatigue is the dominating mechanism in fibre separation. This is promising as progress in this direction may allow more efficient pulping in terms of energy efficiency, fibre quality and stability of the processes at lower energy costs.

Acknowledgements: We acknowledge the Paul Scherrer Institut, Villigen, Switzerland for provision of synchrotron radiation beamtime at the TOMCAT beamline X02DA of the SLS and would like to thank Dr. Borisova for assistance.

Author contributions: All the authors have accepted responsibility for the entire content of this submitted manuscript and approved submission.

Research funding: The Swedish Energy Agency is gratefully acknowledged for financing this work in project 37206-2.

Employment or leadership: None declared. Honorarium: None declared.

\section{References}

Abaqus. (2015) Abaqus 2016 theory guide.

Atack, D., May, W.D. (1958) Frictional mechanisms in the grinding process. Pulp Pap. Mag. Can. Convention issue 265-271.

Atack, D., May, W.D. (1962) Mechanical pulping studies with a model steel wheel. Pulp Paper Mag. Can. 63:T10-T20.
Beath, L.R. (1958) The varying angle between surface and wood - a case of wide, uncontrolled freeness variation in ground wood production. Pulp Paper Mag. Can. 59:123-133.

Björkqvist, T., Tienari, M., Lucander, M. (2007) Simulation of fatigue related variables in wood grinding. Proc. Int. Mech. Pulp. Conf., Minneapolis, MN.

De Magistris, F., Salmén, L. (2005) Deformation of wet wood under combined shear and compression. Wood Sci. Technol. 39:460-471.

De Magistris, F., Salmén, L. (2008) Finite element modelling of wood cell deformation transverse to the fibre axis. Nord. Pulp Pap. Res. J. 23:240-246.

Dornfeld, D.A. (1981) Single grit simulation of the abrasive machining of wood. J. Eng. Ind. 103:1-12.

Dornfeld, D.A., Wu, S.M. (1977) An investigation of ground wood surfaces as related to pulp and stone characteristics. Wear 42:163-175.

Enström, E., Fagerhed, J.-A., Lönnberg, B. (1990) Effects of pulpstone grits in wood grinding. Part I. Distribution of single-size grits. Pap. Puu-Pap. Tim. 72:385-390.

Fortino, S., Hradil, P., Salminen, L.I., De Magistris, F. (2015) A 3D micromechanical study of deformation curves and cell wall stresses in wood under transverse loading. J. Mat. Sci. 50:482-492.

Gåhlin, R., Björkman, H., Rangsten, P., Jacobson, S. (1999) Designed abrasive diamond surfaces. Wear 233-235: 387-394.

Harrington, J.J., Booker, R., Astley, R.J. (1998) Modelling the elastic properties of softwood. Part I: the cell-wall lamellae. Holz. Roh. Werks. 56:37-41.

Heinemann, S., Saharinen, E., Särkilahti, A., Salminen, L.I. (2016) The effect of wood alignment on wood grinding - Part 2: fines character and microscopic observations. Bioresources 11:2526-2536.

Heldin, M., Wiklund, U. (2019) Defibration mechanisms and energy consumption in the grinding zone - a lab scale equipment and method to evaluate groundwood pulping tools. Nord. Pulp. Pap. Res. J. Accepted for publication.

Heldin, M., Isaksson, P., Wiklund, U. (2016) Initiation of wood defibration in groundwood pulping, single asperity indentation and scratching. Nord. Pulp Pap. Res. J. 31:401-406.

Höglund, H., Tistad, G. (1973) Energy uptake by wood in the mechanical pulping process. Proc. Int. Mech. Pulp. Conf., Stockholm, Sweden.

Holmberg, S., Persson, K., Petersson, H. (1999) Nonlinear mechanical behaviour and analysis of wood and fibre materials. Comput. Struct. 72:459-480.

Isaksson, P. (2015) A note on stress fields and crack growth in porous materials subjected to a contact load. Int. J. Solids Struct. 64-65:62-70.

Lucander, M., Björkqvist, T., Touvinen, O. (2010) United States Patent No. US 7,819,149 B2.

Marone, F., Stampanoni, M. (2012) Regridding reconstruction algorithm for real-time tomographic imaging. J. Synchrotron Radiat. 19:1029-1037.

Moilanen, C., Björkqvist, T., Ovaska, M., Koivisto, J., Miksic, A., Engberg, B.A., Salminen, L.I., Saarenrinne, P., Alava, M. (2017) Influence of strain rate, temperature and fatigue on the radial compression behaviour of Norway spruce. Holzforschung 71:505-514. 
Nairn, J. (2006) Numerical simulation of transverse compression and densification in wood. Wood Fiber Sci. 38:576-591.

Reme, P.A. (2000) Some Effects of Wood Characteristics and the Pulping Process on Mechanical Pulp Fibres. Academic dissertation, Trondheim.

Saharinen, E., Särkilathi, A., Salminen, L.I., Heinemann, S. (2016) The effect of wood alignment on wood grinding - Part 1: properties of pulp and fines revealed in the grinding mechanism. Bioresources 11:4201-4211.

Salmén, N.L., Fellers, C. (1981) The fundamentals of energy consumption during viscoelastic and plastic deformation of wood. J. Pulp Paper Sci. 8:TR 93-99.

Salmi, A. What Should a Grindstone Surface Look Like to Produce Pulp with Least Energy? Academic dissertation, Helsinki, 2012.

Salminen, L.I., Liukkonen, S., Alava, M.J. (2014) Ground wood fiber length distributions. Bioresources 9:1168-1178.

Sandås, E. (1991) Effects of pulpstone grits in wood grinding. Part 3. Two-size grit mixture (various sizes). Pap. Puu-Pap. Tim. 73:641-650.
Sandås, E., Lönnberg, B. (1990) Effects of pulpstone grits in wood grinding. Part 2. Two-size grit mixture (various proportions). Pap. Puu-Pap. Tim. 72:765-771.

Tuovinen, O. (2012) United States Patent No. US 8,172,165 B2.

Tuovinen, O. (2016) End of stone age. Proc. Int. Mech. Pulping Conf., Jacksonville, FL.

Tuovinen, O. (2018) Long-term experience with energy-saving mechanical pulping technology. Proc. Int. Mech. Pulping Conf. Proc., Trondheim, Norway.

Tuovinen, O., Fardim, P. (2015) Interrelation between grit morphology and defibration performance in pressurized groundwood process. 0'Papel 76:83-89.

Tuovinen, O., Fardim, P., Wiinamäki, A. (2009) Initial fiber effects in pressurized grinding as analysed by SEM. Proc. Int. Mech. Pulping Conf., Sundsvall, Sweden.

Uhmeier, A., Salmén, L. (1996) Repeated large radial compression of heated spruce, Nord. Pulp Pap. Res. J. 11:171-176.

van Dijk, N., Wu, D., Persson, C., Isaksson, P. (2019) A global digital volume correlation algorithm based on higher-order finite elements: Implementation and evaluation. Int. J. Solids Struct. 168:211-227. 